\title{
Politique
}

\section{Perspectives historiques sur les compressions budgétaires}

\section{James Iain Gow}

Numéro 3, hiver 1983

La crise des finances publiques au Québec

URI : https://id.erudit.org/iderudit/040416ar

DOI : https://doi.org/10.7202/040416ar

Aller au sommaire du numéro

Éditeur(s)

Société québécoise de science politique

ISSN

0711-608X (imprimé)

1918-6584 (numérique)

Découvrir la revue

Citer cet article

Gow, J. I. (1983). Perspectives historiques sur les compressions budgétaires. Politique, (3), 5-25. https://doi.org/10.7202/040416ar d'utilisation que vous pouvez consulter en ligne.

https://apropos.erudit.org/fr/usagers/politique-dutilisation/ 


\title{
Perspectives historiques sur les compressions budgétaires
}

\author{
James Iain Gow \\ Université de Montréal
}

Une lecture de l'histoire de l'administration publique québécoise peut-elle nous aider à éclairer la crise financière que traverse actuellement le gouvernement québécois? Cet éclairage sera diffus, car l'historien pas plus que tous les autres observateurs ne saurait dire avec précision les effets à venir. Néanmoins, l'histoire peut permettre de mieux situer les coupures actuelles dans une longue perspective d'excédents, de déficits et de dettes publiques, d'interventions croissantes de l'État dans la vie économique et sociale ainsi que de centralisation des activités étatiques. Nous allons commenter les cent premières années de cette histoire avant de jeter un regard sur les dix dernières années à la lumière des tendances à long terme retenues.

\section{L'expérience des années 1867-1970}

Deux grandes tendances nous semblent à retenir. D'abord, jusqu'à la fin de la seconde guerre mondiale, les dépenses pouvaient varier de façon absolue tandis que depuis 1949-1950, il n'y a pas eu de baisse en termes absolus (en dollars courants) ni pratiquement en termes relatifs (en dollars constants) ${ }^{1}$. Deu-

1. Nous avons réuni ces chiffres dans Les dépenses du gouvernement du Québec (1867-1970) comme indicateur d'activités étatiques, Note de recherche no 5, Département de science politique, Université de Montréal, janvier 1982. 
xièmement, dans le passé, règle générale, on empruntait pour financer des investissements tandis que seule la faiblesse des revenus justifiait des déficits budgétaires sur le compte ordinaire à l'occasion de conditions économiques difficiles. Commençons par ce second trait.

\section{Surplus et déficits}

$\mathrm{Au}$ tableau 1, nous indiquons les tendances historiques à connaître un excédent ou un déficit sur le compte ordinaire, c'est-à-dire en laissant de côté les dépenses d'immobilisations désignées comme telles. On voit que sauf pendant les périodes de crise financière, la longue période difficile au dernier quart du dix-neuvième siècle et la crise des années 1930, les gouvernements avaient l'habitude de faire un excédent sur le compte ordinaire. Cependant, les gouvernements avaient aussi tendance à jongler avec la comptabilité de sorte qu'à chaque changement de parti au pouvoir, le nouveau gouvernement accusait l'ancien d'avoir caché dans les dépenses extraordinaires ou d'immobilisations des dépenses courantes et ce, afin de dissimuler un déficit ou d'en diminuer l'importance. Certes au cours des années 1930-1935 on a classé comme dépenses extraordinaires des programmes de secours qui ont été reclassifiés par l'Auditeur de la Province vingt ans plus tard, puisqu'elles n'avaient laissé aucune trace tangible en forme d'édifice, de pont, de route, etc. ${ }^{2}$.

En 1970-1971 on a changé la comptabilité du gouvernement du Québec de sorte que la distinction entre les dépenses ordinaires et les dépenses de capital disparaît et, avec elle, les surplus budgétaires auxquels les gouvernements nous avaient habitués. Afin de voir les effets d'une application rétroactive de cette méthode nous avons établi au tableau 2 quel aurait été le surplus ou le déficit encouru depuis $1945 \mathrm{si}$ on avait tenu compte des dépenses de capital. Comme on peut le voir, le

2. Rapport de l'Auditeur de la Province de Québec pour l'année se terminant le 31 mars 1955. 
TABLEAU 1

Tendances historiques de surplus ou de déficit sur les comptes ordinaires

\begin{tabular}{ll}
\hline Surplus & Déficit \\
$1867-1873$ & $1874-1898$ (4 exceptions) \\
$1898-1931$ & $1931-1936$ \\
$1936-1960$ (1 exception) & \\
$1960-1970$ & \\
\hline
\end{tabular}

Sources: Les Comptes publics du Québec relevés par André Bernard, Parliamentary Control of Public Finance in the Province of Québec (thèse MA, McGill, 1964). Annuaire du Québec 1972, p. 813.

conservatisme financier de Maurice Duplessis se traduit par neuf surplus sur le compte global en quinze ans et six déficits, dont deux très petits. Quatre déficits en quinze ans en tenant compte des investissements, c'est un exploit, surtout lorsqu'on se rappelle que c'est sous $M$. Duplessis que la tendance à la hausse des dépenses s'est installée pour de bon. Par contre en tenant compte des dépenses de capital, on voit disparaître les surplus des années 1960 et l'apparition de déficits substantiels.

\section{Coupures passées}

Auparavant, avons-nous dit, les dépenses, comme les revenus étaient plus portés à varier d'une année à l'autre. On a connu des baisses de dépenses, surtout au dix-neuvième siècle (douze fois entre 1875 et 1895), mais aussi pendant les deux guerres mondiales et brièvement (trois fois) pendant la crise des années 1930. Toutes les dépenses pouvaient varier mais certaines le faisaient de façon remarquable. Au dix-neuvième siècle, les chapitres les plus variables sont les subventions aux chemins de fer, le service de la dette, les travaux publics et la colonisation. $\mathrm{Au}$ vingtième siècle ce sont les travaux publics, la voirie, la colonisation et les subventions à l'enseignement supérieur. À l'exception de la dette publique, ces dépenses ont une caractéristique commune: elles sont faites par des personnes autres que les employés de l'État, que ce soient les contracteurs, les sociétés de 
TABLEAU 2

Revenus et dépenses du Gouvernement du Québec, 1945-1946 à 1969-1970

\begin{tabular}{|c|c|c|c|c|c|}
\hline \multicolumn{2}{|c|}{$\begin{array}{l}\text { Revenus } \\
\text { ordinaires }\end{array}$} & \multirow{2}{*}{$\begin{array}{c}\begin{array}{c}\text { Dépenses } \\
\text { ordinaires }\end{array} \\
95,6\end{array}$} & \multirow{2}{*}{$\begin{array}{c}\begin{array}{c}\text { Surplus } \\
\text { ou déficit } \\
\text { ordinaire } \\
\text { en millions } \$\end{array} \\
14,8\end{array}$} & \multirow{2}{*}{$\begin{array}{c}\begin{array}{c}\text { Immobili- } \\
\text { sations }\end{array} \\
13,0\end{array}$} & \multirow{2}{*}{$\begin{array}{r}\begin{array}{c}\text { Surplus } \\
\text { ou déficit } \\
\text { global }\end{array} \\
1,8\end{array}$} \\
\hline $1945-1946$ & 110,3 & & & & \\
\hline 1946- 1947 & 133,4 & 106,9 & 26,5 & 24,3 & 2,2 \\
\hline $1947-1948$ & 167,8 & 123,4 & 44,4 & 40,9 & 3,4 \\
\hline 1948- 1949 & 194,5 & 160,7 & 33,8 & 64,8 & $-31,0$ \\
\hline $1949-1950$ & 207,2 & 175,5 & 31,8 & 22,7 & 9,1 \\
\hline $1950-1951$ & 238,7 & 200,7 & 38,0 & 33,8 & 4,2 \\
\hline $1951-1952$ & 275,7 & 213,6 & 62,1 & 60,3 & 1,8 \\
\hline $1952-1953$ & 283,6 & 254,7 & 28,9 & 67,0 & $-38,1$ \\
\hline 1953-1954 & 297,8 & 261,4 & 36,5 & 58,1 & $-21,6$ \\
\hline $1954-1955$ & 335,1 & 298,4 & 36,6 & 57,9 & $-21,3$ \\
\hline $1955-1956$ & 407,8 & 330,9 & 76,9 & 76,6 & 0,3 \\
\hline 1956- 1957 & 441,4 & 355,2 & 86,2 & 87,5 & $-1,3$ \\
\hline 1957- 1958 & 503,6 & 417,0 & 86,6 & 86,4 & 0,3 \\
\hline 1958- 1959 & 546,9 & 453,0 & 93,8 & 93,4 & 0,4 \\
\hline 1959- 1960 & 598,1 & 489,6 & 108,5 & 108,8 & $-0,3$ \\
\hline 1960-1961 & 636,6 & 618,7 & 17,9 & 126,8 & $-108,9$ \\
\hline 1961- 1962 & 754,1 & 713,6 & 40,5 & 103,3 & $-62,8$ \\
\hline $1962-1963$ & 851,4 & 808,6 & 42,8 & 126,0 & $-83,2$ \\
\hline 1963-1964 & 941,4 & 905,8 & 35,6 & 161,1 & $-125,5$ \\
\hline $1964-1965$ & 1227,7 & 1188,9 & 38,8 & 203,4 & $-164,7$ \\
\hline $1965-1966$ & 1601,6 & 1571,9 & 29,7 & 249,5 & $-219,8$ \\
\hline 1966- 1967 & 1899,6 & 1838,2 & 61,4 & 255,3 & $-193,9$ \\
\hline 1967-1968 & 2314,7 & 2217,7 & 97,0 & 247,6 & $-150,6$ \\
\hline 1968- 1969 & 2662,3 & 2544,6 & 117,7 & 225,9 & $-108,2$ \\
\hline 1969- 1970 & 3004,0 & 2979,9 & 24,1 & 254,9 & $-230,8$ \\
\hline
\end{tabular}

Sources: Annuaire du Québec, 1968-1969, p. 771 et 1972, p. 813.

colonisation ou les universités. À vrai dire, il y a eu peu de coupures dans la petite fonction publique provinciale, à quelques exceptions notables comme l'abolition de la police provinciale entre 1878 et $1884^{3}$. L'une des raisons importantes de

3. Voir notre article «L'administration québécoise de 1867 à 1900: un État en formation", Revue canadienne de science politique, vol. XI , no 3 (septembre 1979), 575-577. 
cette stabilité de la fonction publique est la conception des emplois comme une forme d'aide sociale qu'on devait éviter de retirer si possible. Et ce qui la rendait possible, c'était le fort degré de décentralisation qui signifiait que même parmi les interventions limitées de l'époque, l'administration agissait souvent, comme nous l'avons vu, par personnes interposées.

\section{Changement des activités étatiques}

Au cours des années 1920, s'amorce un changement dans les champs de dépenses ainsi que dans la nature des activités, changements destinés à caractériser l'administration publique québécoise jusqu'à nos jours. Avec la Loi de l'assistance publique de 1921, on rompt avec une très longue tradition de charité privée et religieuse. Désormais le coût de l'entretien des indigents dans les institutions de charité sera partagé par les institutions, les municipalités et le gouvernement provincial. En 1928 est adoptée la Loi des unités sanitaires accordant pour la première fois un rôle d'éducation et d'inspection sanitaire à des fonctionnaires provinciaux en poste sur le terrain. Ensuite, avec la crise, l'État est impliqué dans l'octroi de secours directs massifs, opération qui est cependant, temporaire. Après 1945, c'est la montée des dépenses de l'éducation avec le résultat que, depuis quinze ans les dépenses des affaires sociales et les dépenses de l'éducation consomment près de soixante pour cent de l'ensemble.

La nature des activités change aussi. Bon nombre des grandes interventions étatiques se sont faites aux dépens des municipalités, notamment dans les secteurs de la voirie, du travail, de l'aide sociale et de la santé publique. Les secteurs d'activité dominés par l'Église, notamment la charité, les hôpitaux, la colonisation et l'éducation connaissent le même processus, mais avec un certain retard. Au cours des années soixante, l'État domine dans tous ces domaines, il les contrôle par une règlementation contraignante, dont le fondement est le contrôle budgétaire. Cette centralisation reçoit un puissant renfort par l'instau- 
ration, en 1964, d'un régime de négociation collective des conditions de travail dans le secteur public suivi par la fonction publique en 1965 .

\section{Changement des sources de revenus}

Une dernière remarque sur le long terme. Les sources de revenus de l'État au Québec ont changé de manière appréciable. De 1867 à 1910, les deux sources dominantes sont le subside du gouvernement fédéral et les revenus des terres et forêts qui rapportent toujours au moins un quart et un cinquième respectivement. En 1930 les revenus proviennent de nouvelles sources importantes dont les taxes sur les successions et sur l'essence et les profits de la Commission des liqueurs. Les taxes les plus importantes aujourd'hui, l'impôt sur le revenu des particuliers et la taxe de vente, sont introduites tardivement soit en 1954 et en 1940 respectivement ${ }^{4}$. Tout cela pour rappeler que le profil des revenus d'aujourd'hui avec un tiers provenant de l'impôt sur le revenu des particuliers, un quart du gouvernement fédéral et un cinquième des taxes à la consommation, est apparu récemment. Il est, par ailleurs, plus contraignant pour les gouvernements puisque la recherche de nouveaux revenus chez les uns et la défense de revenus existants chez les autres impliquent des enjeux politiques plus précieux qu'une variation autrefois dans les revenus des terres et forêts ou l'introduction d'une taxe sur l'essence 5 .

\section{Un éclairage sur les dix dernières années}

Afin de jauger les développements les plus récents, on a besoin de séries de chiffres fiables. Étant donné la difficulté

4. L'impôt sur le revenu personnel est, à vrai dire introduit en 1940, mais il est cédé à Ottawa en 1942 pour faire face aux problèmes de la guerre. Seul le Québec créera son impôt du genre dans l'après-guerre.

5. La Commission des liqueurs est née dans la controverse, mais celle-ci portait surtout sur la tempérance, non sur la collecte de revenus. 
d'obtenir des séries absolument comparables qui nous ramènent jusqu'à aujourd'hui, nous utilisons deux tableaux, soit le tableau 3 qui donne les revenus bruts et les dépenses brutes du gouvernement québécois de 1966 à 1979 et le tableau 4 qui donne les recettes et les déboursés de 1977 à 1981. Ce dernier tableau a l'avantage de nous rappeler l'existence d'opérations non budgétaires qui peuvent diminuer ou augmenter les besoins financiers du gouvernement.

Avant de commenter ces données à la lumière de cent ans d'histoire, retenons deux détails qui dégonflent les séries dans leur croissance phénoménale. D'abord nous avons déjà dit que c'est un changement dans la comptabilité en 1970-1971 qui a

TABLEAU 3

Revenus bruts, dépenses brutes et dépenses d'immobilisations 1966-1967 à 1978-1979

\begin{tabular}{cccccc}
\hline \multicolumn{2}{c}{$\begin{array}{c}\text { Revenus } \\
\text { bruts } \\
\text { en millions }\end{array}$} & $\begin{array}{c}\text { Dépenses } \\
\text { brutes }\end{array}$ & $\begin{array}{c}\text { Surplus } \\
\text { ou déficit } \\
\text { global }\end{array}$ & $\begin{array}{c}\text { Immobili- } \\
\text { sation }\end{array}$ & $\begin{array}{c}\text { Surplus } \\
\text { ou déficit } \\
\text { ordinaire* }\end{array}$ \\
\hline 1966-1967 & 2117,2 & 2346,4 & $-229,2$ & 344,3 & 115,1 \\
$1967-1968$ & 2534,3 & 2725,2 & $-190,9$ & 292,5 & 101,6 \\
$1968-1969$ & 2884,0 & 2995,3 & $-111,3$ & 252,5 & 139,2 \\
$1969-1970$ & 3209,3 & 3442,4 & $-233,1$ & 275,0 & 41,9 \\
$1970-1971$ & 3806,9 & 3928,9 & $-122,0$ & 315,9 & 193,9 \\
$1971-1972$ & 4226,9 & 4575,8 & $-348,9$ & 445,4 & 96,5 \\
$1972-1973$ & 4732,5 & 5055,1 & $-322,6$ & 461,3 & 138,7 \\
$1973-1974$ & 5440,4 & 5698,1 & $-257,7$ & 514,5 & 256,8 \\
$1974-1975$ & 6921,5 & 7208,6 & $-287,1$ & 620,0 & 332,9 \\
$1975-1976$ & 7917,7 & $8,791,1$ & $-873,4$ & 747,4 & $-126,0$ \\
$1976-1977$ & 9217,3 & 10208,4 & $-991,1$ & 589,9 & $-401,2$ \\
$1977-1978$ & 11168,3 & 12052,4 & $-884,1$ & 587,8 & $-296,3$ \\
$1978-1979$ & 11928,3 & 13402,8 & $-1474,5$ & 585,9 & $-888,6$ \\
\hline
\end{tabular}

*N.B.: Il s'agit d'une opération hypothétique puisque la distinction entre dépenses ordinaires et autres n'existe plus depuis 1970-1971. Nous avons simplement déduit les dépenses des immobilisations du déficit global.

Source: Annuaire du Québec 1977-1978, p. 1136 et 1979-1980, p. 919. 
créé l'apparition de déficits ininterrompus après trente ans d'excédents. Nous y reviendrons. Ensuite, une bonne partie de la croissance des dépenses est attribuable à l'inflation. Un joli graphique en couleurs de l'Annuaire du Québec 1979-1980 (p. 925) montre que pour les dix ans de 1969-1970 à 1978-1979 inclusivement l'inflation a doublé la croissance réelle des dépenses, c'est-à-dire qu'en dollars constants les dépenses ont presque doublé tandis qu'en dollars courant elles ont presque quadruplé.

\section{TABLEAU 4}

État sommaire des opérations financières sur la base des recettes et déboursés (en millions de dollars)

1. Opérations budgétaires

Recettes

Déboursés

$+11515,9+12724,6+13800,7+15373,0$

$-12465,7-13782,1-15100,9-17767,0$

Déficit $-949,8-1057,5-1300,2-2394,0$

\begin{tabular}{lrrrr} 
2. Opérations non budgétaires ${ }^{1}$ & & & \\
Placements prêts et avances & $-266,1$ & $-270,5$ & $-217,2$ & $-81,0$ \\
Compte des régimes de retraite & $+18,9$ & $+7,3$ & $+75,1$ & $+142,0$ \\
Autres comptes & $+22,0$ & $+55,8$ & $+70,1$ & $-12,0$ \\
& & & & \\
\hline Solde & $-225,2$ & $-207,4$ & $-72,0$ & $+49,0$ \\
\hline
\end{tabular}

3. Besoins financiers nets

$$
-1175,0-1264,9-1372,2-2345,0
$$

${ }^{1}$ Ecarts net entre les recettes et les déboursés.

N.B.: Le signe (+) signifie une source de financement et le signe (-) un besoin de financement.

Source: Gouvernement du Québec, Budget 1982-1983, Renseignements supplémentaires. Crédits. Annexe II, p. 9. 
Nous utilisons ici les chiffres courants, mais nous essayons d'éviter les problèmes dus uniquement à l'inflation par le moyen d'autres comparaisons.

\section{Importance des déficits}

Après ces mises en garde examinons les déficits de ces dernières années. Si on avait gardé la comptabilité traditionnelle, les déficits sur le compte ordinaire n'auraient commencé qu'en 1975-1976. Par contre, si on avait appliqué la comptabilité nouvelle aux gouvernements d'après-guerre la série de déficits sur le compte global commence très exactement avec la Révolution tranquille, en 1960-1961 (tableau 2). Aussi, si on prend une autre mesure, à savoir l'importance du déficit global en termes des revenus ordinaires, on retrouve la Révolution tranquille comme facteur important. Puisque nos tableaux ne se comparent pas dans le détail gardons-nous d'en tirer des conclusions trop précises. Néanmoins, les déficits exprimés en pourcentages des revenus ordinaires sont à peu près les suivants: 1960-1961 à 1965-1966, 12,5\%; 1966-1967 à 1969-1970, un peu plus de $7 \%$; 1970-1971 à 1975-1976, 7\%; et 1976-1977 à 1980-1981, un peu plus de $10 \%$. Évidemment, ce qui inquiète dans les déficits récents, c'est leur montée si rapide. Le déficit de 1981-1982 fut de 2.9\$ milliards, soit $16.5 \%$ des revenus ${ }^{6}$.

\section{Le service de la dette}

Prenons une autre mesure de l'importance de la dette, la part du service de la dette dans l'ensemble. Encore là, la situation jusqu'à très récemment n'avait rien d'alarmant. Dans le passé la situation a été bien pire. Au cours des années 1890 , le service de la dette prenait à lui seul le tiers des dépenses totales

6. Jacques Parizeau, Budget 1981-1982. Énoncé complémentaire aux politiques budgétaires du gouvernement, le 17 novembre 1981, 2. et la Presse, 13 novembre 1982, B- 1 . 
et parfois $40 \%$ des dépenses ordinaires. Le conservatisme financier des gouvernements Libéraux des premières trente-cinq années de ce siècle ainsi que celui de M. Duplessis de 1944 à 1959 a graduellement réduit l'importance du service de la dette parmi les dépenses nettes à 3,9\% en 1959. Elle augmente un peu au milieu des années 1960 mais grâce à la prudence financière de l'union Nationale, elle baisse à nouveau à 3,7\% en 1969. Nos chiffres pour les années les plus récentes concernent les dépenses brutes: on y voit une progression constante dans l'importance du service de la dette. De 4,9\% en 1976- 1977 elle passe à 7,0\% en 1980-1981 et $\mathbf{M}$. Bérubé prévoit qu'elle sera de $8,6 \%$ en $1982-1983^{7}$. On est encore loin des proportions alarmantes du $19 e$ siècle comme on est encore loin des $22 \%$ des dépenses fédérales qui seront allouées cette année au service de la dette du Canada. Ce qui inquiète c'est que le service de la dette québécoise a augmenté de près de $40 \%$ au cours des deux dernières années et si les efforts de M. Bérubé ne sont pas couronnées de succès on pourrait se trouver très rapidement dans la même position que le gouvernement fédéral ${ }^{8}$.

\section{La part du PIB}

Une dernière mesure de la situation évoque un critère ignoré jusqu'ici: la part des dépenses de l'État dans l'économie. Pour les années avant 1970, nous l'avons exprimée au tableau 5 en pourcentage du produit national brut. Depuis cette date on utilise le produit intérieur brut qu'on voit, pour les dernières années au graphique 1. Encore là la croissance des années 1960 est bien plus forte que celle des années 1970: la part de l'Etat double de 1960 à 1970, tandis qu'elle n'augmente que d'un

7. Annuaire du Québec 1979-1980, Conseil du Trésor, Budget, 1982-1983, Renseignements supplémentaires, Crédits, le 23 mars 1982, 53.

8. Pierre Fortin et Pierre Fréchette, Les défis du développement socio-économique du Québec dans les années 1980, Québec, Conseil de planification et de développement du Québec, novembre 1981, 5. 
TABLEAU 5

Dépenses totales d'activité en pourcentage du produit national brut

\begin{tabular}{ccccc}
\hline & $\begin{array}{c}\text { Dépenses } \\
\text { totales } \\
\text { d'activité } \\
\$ 000000\end{array}$ & Année & $\begin{array}{c}\text { Produit } \\
\text { national } \\
\text { brut } \\
\$ 000000\end{array}$ & $\begin{array}{c}\text { Dépenses en } \\
\text { pourcentage du } \\
\text { produit national } \\
\text { brut }\end{array}$ \\
\hline $1946-1947$ & 121,4 & 1946 & 3006 & 4,03 \\
$1950-1951$ & 223,2 & 1950 & 4574 & 4,87 \\
$1955-1956$ & 392,3 & 1955 & 6705 & 5,85 \\
$1960-1961$ & 736,5 & 1960 & 9178 & 8,02 \\
$1965-1966$ & 837,7 & 1965 & 13226 & 13,89 \\
$1970-1971$ & 3648,7 & 1970 & 21136 & 17,26 \\
\hline
\end{tabular}

Sources: Note de recherche no 1, Tableau type des comptes économiques du Québec 19461968, Québec, Ministère de l'industrie et du Commerce, 1968, Statistiques, vol. IX, no 4 (1971) et vol. XI, no 4 (1973).

cinquième environ de 1970 à 1980. Deux choses dérangent aujourd'hui. D'une part, après avoir plafonné autour de $24 \%$ de 1977 à 1980, le pourcentage du produit intérieur brut consommé par les dépenses québécoises s'est mis à monter à nouveau pour atteindre probablement $27 \%$ en 1982-1983. D'autre part ce sont les effets cumulatifs des interventions passées et celles du gouvernement fédéral qui rendent la situation sérieuse.

Lorsqu'on tient compte des dépenses du gouvernement fédéral et de celles des gouvernements locaux on peut dire que les dépenses du secteur public au Québec atteignent 47,4\% du produit intérieur brut en $1980^{9}$.

Ce qui agace ici c'est que même avec des coupures budgétaires on ne semble pas capable de restreindre la croissance des dépenses en deça de la croissance du produit intérieur brut ou

9. Bureau de la statistique du Québec, Comptes économiques des revenus et des dépenses. Québec, 1961-1980, 1982, 17. 


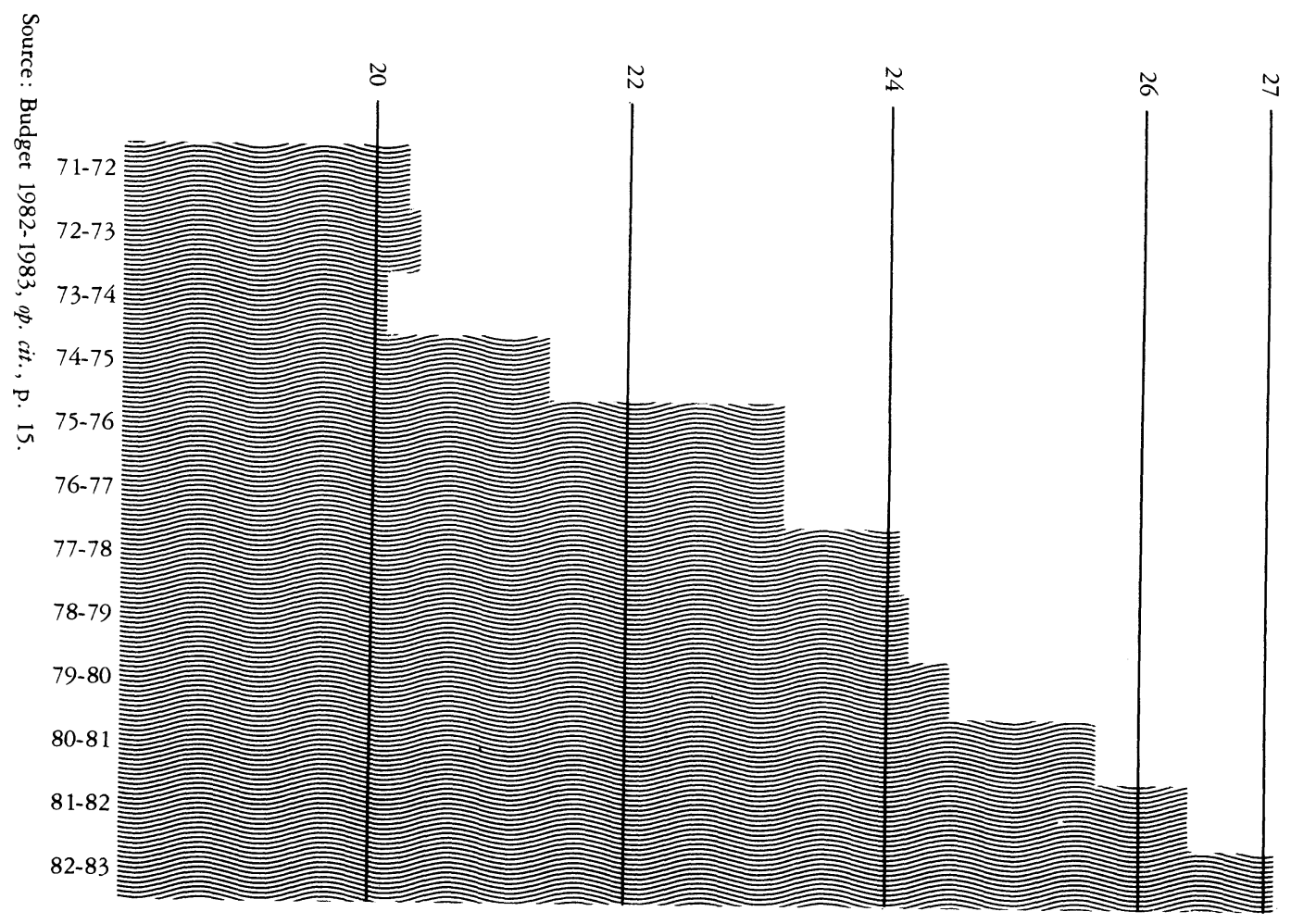

a

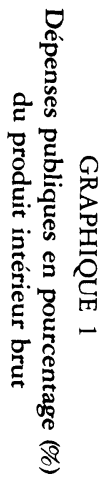


encore de celle de l'indice des prix à la consommation. Ainsi dix ans après que Gérard Bélanger ait parlé du drame pénible «d'un budger croissant pour des services constants ${ }^{10}$, il semble qu'on ait toujours des budgets croissants pour des services décroissants du moins dans les grands secteurs de dépenses comme l'éducation et les affaires sociales.

\section{Les problèmes de l'État providence}

Comme nous l'avons fait dans les parties précédentes de ce texte, nous désirons commenter la situation actuelle dans une perspective à moyen sinon à long terme plutôt que de nous prononcer sur l'un ou l'autre aspect de nos controverses. Nous développerons donc trois idées touchant d'abord les limites que semble avoir atteintes un certain type d'État, ensuite la logique ou la rationnalité qui fonde nos décisions et finalement les rapports entre la centralisation et la décentralisation.

\section{Les limites d'un certain type d'État}

Nous semblons avoir atteint les limites d'un certain type d'État, un État-providence centralisé qui tend vers l'égalité uniforme. Des limites semblent atteintes sur le plan global car, rendu à $47 \%$ du produit intérieur brut, l'État ne peut guère prendre plus de place sans entrer plus directement dans le champ économique. Déjà il a le monopole ou presque dans les domaines de l'éducation, de la santé et de l'aide sociale. Sans doute l'entreprise privée trouvera d'autres «externalités» dont les frais doivent être assumés par les contribuables mais à moins d'entrer plus directement dans le champ économique il semble difficile que l'État aille beaucoup plus loin ${ }^{11}$. De toute façon on

10. Gérard Bélanger, «Le service public: un budget croissant pour des services constants" dans J.-L. Migué (sous la direction de), Le Québec d'aujourd'bui - regards d'universitaires, Montréal, HMH Hurtubise, 1971, 17.

11. Jean-P. Vézina, "L'évolution des dépenses et recettes des administrations publiques. La poursuite du scénario actuel est-elle possible?", Actualité économique, $53^{\mathrm{e}}$ année (1977), 413. Voir aussi Pierre Rosanvallon La crise de l'État-providence, Paris, Éditions du Seuil, 1981, ch. 1 et 2. 
ne voit aucun parti important au Québec qui prône une socialisation accrue des moyens de production. Et d'une manière ou de l'autre l'État va devoir être plus attentif à l'économie de ses moyens.

Les limites, cependant, ne sont pas que financières et économiques. Le type d'État que nous avons créé depuis vingt ans met à l'épreuve notre système de démocratie représentative. Depuis plusieurs années les observateurs et les intéressés euxmêmes constatent l'impossibilité de donner un sens pratique à la responsabilité ministérielle. Il est matériellement impossible à un ministre fut-il entouré de l'équipe la plus brillante, d'être responsable de la conduite des centaines ou des milliers de fonctionnaires qui travaillent en principe sous ses ordres. C'est une question de temps et d'attention. Accaparé par des préoccupations politiques et parlementaires, il ne peut consacrer qu'une partie de son temps et de son attention à toutes les questions d'administration courante qui façonnent l'État à la longue. D'ailleurs le sous-ministre n'est guère mieux placé, seul à la tête de la pyramide. Même si les postes de sous-ministre adjoint ont connu la croissance la plus rapide de toutes les catégories d'emploi ces dernières années, nous restons avec l'impression d'un surfait de cadres supérieurs et d'une administration incontrôlée. La situation n'est guère plus satisfaisante du côté de la législature et des partis politiques. Avec tous les enjeux, avec toutes les activités qui concernent ses électeurs, un député a beaucoup de mal à représenter de 30000 à 40000 personnes auprès de la législature, du gouvernement et de l'administration. Les propositions de réforme du mode de scrutin ne pourront pas améliorer cette situation, car toute variante de la proportionnelle ajoutera des représentants des partis, non des électeurs comme tels. Même les partis à l'échelle du Québec ont du mal à intégrer les intérêts si divers de leurs membres. Le Parti Québécois est celui qui a le plus essayé d'assurer une participation populaire, mais lors de son congrès du mois de décembre 1981 et avant les bouleversements que l'on sait, il se proposait d'étudier en une fin de semaine plus de 2000 propositions provenant des associa- 
tions locales. L'idée à retenir dans cette discussion c'est que les citoyens membres du parti avaient beaucoup de questions et de problèmes sur le cœur même si le congrès biennal n'était pas l'endroit où ils pouvaient être tous discutés.

Ces remarques sur l'impossible responsabilité ministérielle et les difficultés des citoyens de se faire représenter au sein des institutions nationales suggèrent qu'il faut chercher une solution dans un rapprochement des services et des citoyens. Avant d'aborder cette question examinons un instant la logique du gouvernement à l'heure des coupures.

\section{La rationalité de l'État}

Il est utile de se demander avec quelle logique ou rationalité on a pris les décisions politico-administratives des vingt dernières années et quelle est celle qui prévaut aujourd'hui. Il y a évidemment la logique des rapports de force qui détermine ce qui est faisable en politique. Il n'est pas nécessaire de faire ici la démonstration de l'influence des grandes entreprises dans ce jeu. Depuis longtemps, elles font connaître leurs besoins et leurs exigences aux gouvernements, aujourd'hui elles sont simplement moins nombreuses et plus fortes que jamais. Ce qui est nouveau depuis quinze ans c'est que l'État a accepté de négocier les conditions de travail et de carrière avec les employés de la fonction publique et du secteur public. On considère aujourd'hui que sa marge de manœuvre est considérablement réduite par des concessions faites dans le passé récent aux chapitres des salaires et avantages comme à celle de la sécurité d'emploi. Quelle que soit l'issue de l'impasse provoquée par la loi 70, le gouvernement actuel devra arriver à une sorte d'entente nouvelle avec ces syndicats. Ce qui frappe, c'est que ceux qui ont le plus de poids auprès des gouvernements, entreprises comme syndicats, sont les producteurs. Face aux coupures il y a danger que l'intérêt de la clientèle soit négligé. 
A propos de clientèle, on peut se demander dans quelle mesure les services gouvernementaux suivent une logique de marché. Comme aiment à nous le rappeler les tenants du Public Choice, le marché joue très peu dans les administrations publiques autres que les quelques entreprises commerciales et industrielles qui ont le mandat d'être rentables. Il y a cependant un autre marché, le marché politique, et certains observateurs ont identifié un marché à très court terme qui joue juste avant les élections ${ }^{12}$. Deux choses nous semblent importantes dans le contexte de notre discussion: les citoyens ont semble-t-il la mémoire très courte, de sorte qu'ils donnent raison aux gouvernements dans leurs visions à très court terme. Aussi, les gouvernements dans leur programme-propagande-marketing parlent d'habitude moins des revenus que des dépenses. Donc s'il y a un marché politique à court terme, c'est un marché de carte de crédit, acheter maintenant, payer plus tard.

Il y a, cependant, une autre logique qui est devenue très importante au cours des vingt dernières années. C'est la logique de la rationnalité bureaucratique ou de la planification administrative. C'est elle qui nous a donné le régime des rentes, la Caisse de dépôts, la régionalisation scolaire, le réseau des affaires sociales. Ce n'est ni une logique politique des rapports de force, ni une logique de marché, c'est une logique calculatrice qui se base sur différentes estimations des besoins de la population et des coûts inhérents aux projets, estimations qui sont faites par les technocrates de l'État avec ou sans le concours d'expertises externes. Le test de cette logique, c'est de persuader les ministres d'une chose, les fonctionnaires n'ayant à assumer ni les frais économiques ni les coûts politiques de leurs gestes. C'est inévitable et ce n'est pas un défaut mais on peut trouver curieux

12. André Blais fait un relevé des écrits à l'appui de cette hypothèse qu'il fait sienne dans «Politique agricole et résultats électoraux au Québec", dans la Revue canadienne de science politique, vol. XI, no 2 (juin 1978), 378-381. Voir aussi notre note de recherche (cf. note 1) aux pages 36-39. aussi Vincent Lemieux, "Les plates-formes électorales des partis», dans Quatre élections provinciales au Québec, 1956-1966, Québec, P.U.L., 1969, 60. 
qu'en cas de pépin ou de difficulté de réalisation des projets, l'on confie aux fonctionnaires le soin de trouver des solutions de rechange. Depuis vingt ans, la logique administrative nous a donné des systèmes toujours plus complexes. C'est une logique plutôt rationnelle qu'expérimentale. Il peut y avoir des expériences mais le plus tôt possible on s'empresse d'installer un systèmes national, avec politiques et normes établies dans la capitale. ${ }^{13}$

Cette logique était plus appropriée à une période de croissance que de crise. Elle était aussi un peu arrogante: on disait vouloir rattraper vingt, trente ou bien cinquante ans de retard sans admettre que c'était grâce au conservatisme financier des gouvernements passés qu'on pouvait se lancer dans des investissements importants sans trop de soucis.

\section{La nécessaire décentralisation}

Il est tellement banal d'arriver à une conclusion pareille que nous aimerions montrer en quoi elle est liée à la question des coupures et à l'analyse qu'on vient de faire des origines plus ou moins récentes de notre situation actuelle. D'abord la centralisation que nous avons vécue depuis 1960 était en bonne partie nécessaire. Les systèmes décentralisés des temps passés (municipal, scolaire, de santé et d'affaires sociales) furent mal organisés, éparpillés en petites unités sans ressources financières adéquates, apathiques, dominés par l'esprit de clocher et des notables traditionnels. Le tout se soldait par une inégalité des services disponibles aux citoyens. Aujourd'hui les problèmes semblent différents. Le gouvernement trouve son compte en déléguant aux directions des établissements d'enseignement et des affaires sociales le pouvoir de choisir ce qu'il faut couper. Mais peut-on aller plus loin et voir en quoi une plus grande décentralisation serait appropriée, désirable et faisable?

13. Voir Marc Renaud «Réforme ou illusion? Une analyse des interventions de l'État québécois dans le domaine de la santé», Sociologie et sociétés, IX, 1 (avril 1977) 127-129. 
Il semble bien que nos problèmes aujourd'hui soient aggravés par le fait que plusieurs grandes tendances exigent notre attention en même temps alors que jadis on pouvait s'y attaquer à tour de rôle. Le chômage, l'inflation, l'énergie, la pollution, le crime, tous ces problèmes réclament des interventions, mais nous sommes un peu comme ces malades atteints de plusieurs maladies dont le traitement de l'une aggrave les autres. Pour faire face à tous ces problèmes nous aurons besoin d'innovations et, pour cela, d'expérimentation. L'administration nationale est capable par moments et à certains endroits d'une certaine expérimentation mais ce n'est pas là sa tendance dominante. Pour sortir de l'uniformité paralysante, il nous semble qu'on a besoin de centres de compétence qui peuvent rivaliser avec la fonction publique provinciale ${ }^{14}$. Ainsi ce n'est pas en affaiblissant les commissions scolaires qu'on encouragera l'innovation pédagogique et scolaire.

Une décentralisation accrue pourrait faciliter non seulement l'innovation, mais aussi une résolution partielle des dilemmes de représentation et de responsabilité politique déjà évoqués. Sur ce plan, nous n'avons pas de foi aveugle dans la démocratie locale; elle peut tourner à la corruption politique et il est prévisible qu'elle se bureaucratisera à son tour. Nous ne croyons pas non plus à une participation communautaire soutenue de longue durée dans les affaires de la ville. Par contre, les fonctionnaires locaux sont accessibles et le gouvernement local permet aux élans populaires de trouver un moyen d'intervention avec un minimum de mobilisation de ressources. En tout cas c'est seulement en plaçant les fonctionnaires dans des situations de responsabilité partagée entre les sommets politiques et administratifs et la clientèle qu'on trouvera une solution au problème de la responsabilité ministérielle fictive. Ceci est plus important que jamais dans une période de coupures budgétaires. On n'obtiendra pas ainsi la logique du marché mais on fera fléchir peut-

14. Voir Michel Crozier, «La crise bureaucratique» dans la Revue française d'administration publique, no. 15, (juillet-septembre 1980), 593-604. 
être la logique administrative et on donnera plus de poids politique aux citoyens peu ou mal organisés.

Il y a un autre argument qui rend souhaitable dans le contexte actuel une plus grande décentralisation. La force du grand État bureaucratique centralisé est en bonne partie illusoire. Elle semble acquérir une autonomie vis-à-vis son environnement, mais elle est rigide (bloquée dirait Michel Crozier) et elle est vulnérable. Le mouvement «survivalist» qui se développe aux États-Unis peut choquer ou faire sourire selon les réactions de chacun, mais la décision de se bâtir un abri et d'y stocker des vivres suffisants pour six mois ou un an d'entretien d'une famille ne reflète pas seulement la peur d'une guerre thermonucléaire. Elle est aussi une manifestation d'un manque de confiance dans la capacité des gouvernements de maintenir l'ordre civil dans l'éventualité de nouvelles crises. Il n'est pas question d'approfondir cela ici, mais il nous faut rappeler la faiblesse d'un État qui centralise trop ses négociations collectives, son système d'analyse économique, ses banques de données, sa politique énergétique, sa politique culturelle, etc. On peut soutenir que même avec des chevauchements et des doubles emplois un État décentralisé est plus fort, plus résistant que l'autre.

Est-ce que ce sont des spéculations inutiles, des choses qui risquent de ne pas se produire dans cette période de coupures budgétaires? Certes, le gouvernement actuel est plutôt interventionniste et centralisateur que décentralisateur et ce, malgré la réforme de la fiscalité municipale et la création des municipalités régionales de comté. Il a cependant, manifesté un intérêt pour une décentralisation plus poussée et, encore une fois, il pourrait y trouver son compte dans une période de compressions budgétaires. Un regroupement des services municipaux, d'affaires sociales et scolaires sous un gouvernement local ou régional permettrait pour le moins de trouver des solutions à plusieurs problèmes persistants. En effet, il faut mettre fin au scandale des élections scolaires et sociales avec leurs taux de participation dé- 
risoires. Il semble bien que le public vote là où il sent qu'il y a un enjeu d'importance. Un gouvernement local unique aurait plus de chance d'attirer l'intérêt des électeurs. De plus les divisions actuelles empêchent l'utilisation rationnelle des espaces et des équipements. Le problème de la réaffectation des écoles fermées ou de l'utilisation des cafétérias d'école pour offrir des repas bon marché aux personnes âgées, ce genre de problème aurait beaucoup moins d'acuité si on n'avait pas à faire face à trois réseaux d'institutions juridiquement autonomes.

Il ne faut pas se faire trop d'illusion. Le grand État bureaucratique risque d'être avec nous, au-dessus de nous, pour longtemps. Il est le patron de la plupart d'entre nous soit comme employeur soit comme source de largesses et de contraintes. À la longue il n'est pas certain qu'il soit moins arbitraire que le roi-patron d'autrefois. Après plusieurs années d'étude de l'administration publique, nous arrivons à la même conclusion qu'André Gorz ${ }^{15}$ :

Nous savons désormais que la société ne sera jamais «bonne» par son organisation, mais seulement en raison des espaces d'autonomie, d'auto-organisation et de coopération volontaire qu'elle ouvre aux individus.

Ces réflexions s'inspirent donc d'une tentative de placer la crise financière actuelle dans un contexte historique. Cette perspective permet de voir que nous sommes loin d'être aussi mal placés que nos ancêtres du dix-neuvième siècle mais, par contre, nous avons un État d'un type très différent qui ne peut se retirer de la plupart de ses activités sans affecter péniblement ses citoyens ou ses employés. Les origines de cet État-providence remontent aux années 1920, mais c'est surtout depuis 1960 que l'État québécois a adopté un type de comportement qui pouvait fonctionner en temps de croissance mais qui rencontre des limites certaines lorsque ses effets cumulatifs se font sentir en pé-

15. André Gorz, Adieux au prolétariat, Paris, Éditions Galilée. 1980, la citation, tirée de la couverture, abrège une phrase de la p. 175. 
riode de régression. Sans vouloir jouer au prophète nous avons essayé d'établir quelques effets heureux qui pourraient sortir de la crise si on décidait de réaliser les bonnes intentions de décentralisation. 